\title{
ABM Clinical Protocol \#33: Lactation Care for Lesbian, Gay, Bisexual, Transgender, Queer, Questioning, Plus Patients
}

\author{
Rita Lynne Ferri, ${ }^{1}$ Casey Braitsch Rosen-Carole, ${ }^{1-3}$ Jason Jackson, ${ }^{1,2}$ Elizabeth Carreno-Rijo, ${ }^{1,2}$ \\ Katherine Blumoff Greenberg, ${ }^{1-3}$ and the Academy of Breastfeeding Medicine
}

\begin{abstract}
A central goal of The Academy of Breastfeeding Medicine is the development of clinical protocols for managing common medical problems that may impact breastfeeding success. These protocols serve only as guidelines for the care of breastfeeding mothers and infants and do not delineate an exclusive course of treatment or serve as standards of medical care. Variations in treatment may be appropriate according to the needs of an individual patient.
\end{abstract}

\section{Definitions}

Several sources have defined terms related to LGBTQ+ health. In this study, we reference the University of California San Francisco Transgender Care and Treatment Guidelines ${ }^{1}$ and the National LGBT Health Education Center's glossary of terms. ${ }^{2}$ However, it is important to note that terminology is fluid and community specific. In countries speaking languages other than English, these terms may have adaptations, or may be irrelevant entirely. Consulting with members of LGBTQ+ advocacy communities in such areas, where possible, may be helpful to ensure that language is respectful and inclusive.

- LGTBQI+: A term for people who identify as lesbian $(\mathrm{L})$, gay $(\mathrm{G})$, bisexual $(\mathrm{B})$, transgender $(\mathrm{T})$, queer $(\mathrm{Q})$, questioning $(\mathrm{Q})$, and people with other diversities in sexual orientation and gender identity (+). There are a variety of these terms internationally with their own acronyms. This term is meant to be inclusive.

○ Lesbian (adj., noun): A sexual orientation that describes a woman who is emotionally and sexually attracted to other women.

- Gay (adj.): A sexual orientation that describes a person who is emotionally and sexually attracted to people of their own gender. It can be used regardless of gender identity but is more commonly used to describe men.

○ Bisexual (adj.): A sexual orientation that describes a person who is emotionally and sexually attracted to people of their own gender and people of other genders.
- Transgender (adj.): Describes a person whose gender identity and assigned sex at birth do not correspond. Also used as an umbrella term to include gender identities outside of male and female. Sometimes abbreviated as trans.

- Queer (adj.): An umbrella term used by some to describe people whose sexual orientation or gender identity is outside of societal norms. Some people view the term queer as more fluid and inclusive than traditional categories for sexual orientation and gender identity. Owing to its history as a derogatory term, the term queer is not embraced or used by all members of the LGBT community.

○ Questioning (adj.): Describes an individual who is unsure about or is exploring their own sexual orientation and/or gender identity.

O “+”/Plus: The plus sign represents the evergrowing list of terms people use to describe their sexual orientation or gender identity. There are many different variations of the LGBTQ+ acronym, and the "+" acknowledges that it is not possible to list every term people currently use.

- Affirming care: Refers to care that supports a patient's gender identity, and must include inclusive terminology, practices, insurance coverage, and knowledgeable providers.

- Affirmed pronouns and name: Pronouns and name that are chosen by the individual and, therefore, best represent their gender identity. People in the LGBTQ+ community may have changed their name and gender,

\footnotetext{
${ }^{1}$ University of Rochester School of Medicine and Dentistry, Rochester, New York, USA.

Departments of ${ }^{2}$ Pediatrics and ${ }^{3}$ Obstetrics and Gynecology, University of Rochester School of Medicine and Dentistry, Rochester, New York, USA.
} 
informally or legally, to those that affirm their true gender identity.

- Assigned female at birth, assigned male at birth: These terms refer to gender assignment at birth medically and socially, generally based on genital anatomy. These terms may be abbreviated (AFAB, AMAB) to communicate birth anatomy in medical documentation.

- Cisgender: Someone whose gender identity aligns with the gender assigned to them at birth. For example, someone who was AFAB who identifies as a woman.

- Chestfeeding: A term used by many masculineidentified trans people to describe the act of feeding their baby from their chest, regardless of whether they have had chest/top surgery (to alter or remove mammary tissue). ${ }^{3}$

- Colactation: When more than one parent breast/ chestfeeds their child.

- Gender-affirming surgery: Surgeries specific to transgender people include feminizing and masculinizing procedures that align secondary sexual characteristics with a person's gender identity. These may include facial, voice, genital, and hair removal/addition procedures.

- Gender-expansive, genderqueer, nonbinary: All different terms for a broad category of gender identities in which the individual identifies outside of a binary concept of gender (binary meaning "male" and "female"). This can mean identifying as both feminine and masculine, or as neither.

- Gender identity: A person's innate sense of their own gender. It does not necessarily correspond to anatomy, sex assigned at birth, or how someone expresses themselves. Examples include, but are not limited to, cis woman, cis man, trans man, trans woman, nonbinary, gender expansive, and gender "fluid" (as opposed to "fixed"). Not the same as sexual orientation (see hereunder).

- Gender incongruence, formerly "gender dysphoria" or "gender identity disorder": Incongruence between an individual's experienced or expressed gender and their assigned sex. ${ }^{4}$ Dysphoria refers particularly to suffering as a consequence of this incongruence.

- Heteronormative/cisnormative: the assumption and/or preference of individuals and institutions that everyone is heterosexual and cisgender. This leads to invisibility and stigmatization of people in the LGBTQ+ community.

- Transition: The process and time during which a person assumes their affirmed gender expression that may or may not include legal, medical, or surgical components.

- Sexual orientation: The aspect of someone's identity that refers to the gender(s) of the people to whom they are attracted. Examples include, but are not limited to, homosexual, lesbian, gay, heterosexual, bisexual, asexual, and pansexual.

\section{Purpose}

Children raised by LGBTQ+ parents are well adjusted and healthy, and in such families children thrive. ${ }^{5}$ Despite this, people who are transgender and/or whose sexual orientation is not heterosexual frequently experience misunderstanding and stigma, including in medical interactions. ${ }^{6,7}$ Their medical care is often inadequate, ranging from a failure of health care professionals to recognize their unique needs (e.g., in sexual and reproductive health care), to the enforced use of heteronormative procedures (e.g., registering a trans man as a woman in their medical record). ${ }^{6}$ In many regions of the world, families who had been unable to raise children because of their gender identity and/or sexual orientation now have the opportunity to become parents. This is largely due to the liberalization of adoption policies, along with advances in fertility management, surrogacy, and transition-related health care for transgender individuals. Pregnant people and parents who identify as LGBTQ+, therefore, need access to nontraditional lactation supports that may be unfamiliar to health care providers. In particular, barriers to lactating LGBTQ+ individuals include confronting highly gendered assumptions in the world of childbirth, effects of transition (e.g., hormonal, surgical), impacts of induced lactation, colactation, and hospital considerations. The Academy of Breastfeeding Medicine (ABM) seeks to provide guidance to those caring for individuals who identify as LGBTQ+.

\section{Background and the Importance of Language}

When concerned about discrimination in health care settings, patients frequently do not self-disclose their LGBTQ+ identities. $^{6,8}$ Transgender patients, in particular, have a history of experiencing discrimination and even violence in health care settings. ${ }^{9}$ In one U.S.-based study, one-third of transgender individuals reported having at least one negative health care experience. In the same study, nearly one-third of individuals reported that none of their health care providers knew their gender status. ${ }^{6}$ This has implications for delayed diagnosis and treatment, such as the possibility of a missed ovarian torsion or tumor in a transgender man or undiagnosed prostatic disease in a transgender woman. ${ }^{10,11}$ At its most severe, discrimination by health care providers has led to patient deaths from potentially treatable illnesses due to the refusal of providers to care for transgender individuals. ${ }^{12,13}$

Although the terms introduced previously may be unfamiliar to some providers, recognizing and affirming patients' names, pronouns, and family members are a cornerstone of providing affirming care for patients who identify as LGBTQ+. Traditionally, health professional education has contained little to no content related to caring for patients who may be LGBTQ+ identified. As a result, LGBTQ+ communities have been marginalized in health care systems and settings. Experiences of stigma and discrimination, both in and outside of health care contexts, have led to both severe psychological pain and disparate health outcomes. This is most extreme in areas of sexual, reproductive, and mental health. Affirming health care, including using affirming names and pronouns, and recognizing individual patients' families and communities, can help to mitigate the effects of stigma and improve health. ${ }^{14}$

\section{General Considerations}

- Outward appearance may not match gender identity. Do not assume that a female-appearing individual identifies as female, or is interested in breast/chestfeeding. ${ }^{15}$

- Ensure that people are addressed by their affirmed names and pronouns. To know, one needs to ask. 
O Introductions to a patient can include a provider's own pronouns, which may make the patient feel more at ease sharing their own. (e.g., "My name is Dr. X, I use she/her pronouns.",

$\bigcirc$ Predominantly masculine pronouns $=$ he/him $/$ his/ himself

○ Predominantly feminine pronouns $=$ she/her $/$ hers $/$ herself

- Gender-neutral pronouns $=$ they/them/theirs/themselves; Ze/Zir (Hir)/Zirs (Hirs)/Zirself (Hirself).

- Patients may also use different terms for parenting (mom/mum, dad/father, parent, etc.) and lactation (breastfeeding, chestfeeding, lactation, etc.). It is most respectful to ensure that patients have the opportunity to identify which words they would like to use for their visit at the beginning of the visit.

- Misgendering, or calling a patient by a name, pronoun, or parenting term other than their affirmed name/ pronoun, is hurtful to the patient. When done intentionally, it may sever the patient/provider relationship and put the patient's health at risk. When done unintentionally, it is recommended to acknowledge the mistake, correct the pronoun, and continue with the visit using the correct pronouns and name. It is best that the mistake be acknowledged so that the individual feels respected, but prolonged attention on the mistake may take the focus off of providing appropriate and affirming health care.

\section{Creating a Respectful Health Care Environment}

There are many opportunities for health care systems, hospitals, and clinics to provide affirming care to LGBTQ+ individuals. Provider and staff mistakes surrounding an individual's gender or sexual orientation can be minimized with increased training and by having inclusive systems and documentation. For example, intake forms should be updated to include more options for gender, pronouns, and sexual orientation. "Male" and "female" assigned bathrooms may pose a problem for gender-expansive or transgender people. It may force an individual to use a bathroom that is less accessible or unsafe, instead of using one that aligns with their gender identity. Some countries have laws that prevent transgender people from using bathrooms that align with their gender identity, which poses a safety and violence risk for transgender people. ${ }^{16}$ Having gender-neutral bathrooms (or single-user) mitigates these concerns and is an important aspect of respectful care. Displaying signs or statements of inclusivity is a subtle, but impactful way to acknowledge individuals with gender and sexual diversities. ${ }^{17,18}$ Patient confidentiality is another cornerstone to LGBTQ+ care, as family and friends of a patient may not be aware of their gender identity and/or sexual orientation. Disclosing this information may compromise the patient's safety. It is, therefore, important to ask the patient how much you can disclose if you need to talk to other people in their lives. Although not a comprehensive list, other considerations include:

- consideration of the name of the clinic or organization in which people seek care (avoiding gendered terms such as "women's," "maternal," and "moms" in favor of more inclusive terms such as "parent," "prenatal," "pregnancy," "reproductive," or "lactation”),
- displaying affirmed, rather than legal, names and gender on documentation such as printed schedules and patient wristbands,

- avoiding gendered prefixes and pronouns when someone's gender is unknown (e.g., use a patient's given first and last name, or another nongendered culturally specific form instead of Mr./Ms. X).

\section{Effects of Transition-Related Health Care on Pregnancy and Breast/Chestfeeding}

Transgender and gender expansive individuals may seek out gender-affirming medical and/or surgical treatment to help align their physical appearance to their gender identity. These therapies can begin in early adolescence for the purpose of pubertal suppression. Hormone therapy for transgender individuals is typically either masculinizing or feminizing.

- Pubertal suppression and timing of gender-affirming treatments: gonadotropin releasing hormone agonist (GNRHa) therapy may be used in carefully selected early adolescents to temporarily halt the development of secondary sexual characteristics to lessen gender dysphoria and allow time for decisions to be made concerning gender-affirming treatments. The use of GNRHa pauses the development of irreversible secondary sexual characteristics, but also prevents maturation of primary oocytes and spermatogonia. ${ }^{19}$ For adolescents who move from GNRHa therapy on to cross-gender hormones, no current studies address the possibility of future fertility.

For transgender people who want to preserve their fertility and/or breast/chestfeeding capability, deciding when to start gender-affirming treatment can be difficult. For example, delaying gender-affirming femaleto-male chest reconstruction ("top" surgery) for a trans man until after the completion of breast/chestfeeding may prolong his distressing gender dysphoria for many years. ${ }^{3}$ In addition to the risk of continued gender dysphoria, another challenge is the lack of data on the effects of postpubertal hormone therapy on fertility, including the thresholds for causing infertility. ${ }^{19,20}$ Fertility preservation is a growing field, and new methods are being investigated. ${ }^{19}$ Various forms of fertility preservation exist (i.e., testicular sperm extraction and banking, oocyte harvesting and banking, and ovarian tissue cryoprecipitation) and should be discussed with individuals before starting therapies that may affect fertility. ${ }^{19,21,22}$

- Feminizing treatments and practices: Hormone therapy for trans women and transfeminine people typically includes an estrogen and antiandrogen (such as spironolactone), and may also include a progestin. Estrogen therapy will induce breast tissue development. ${ }^{21,23,24}$ There is one case report and many anecdotal reports of trans women inducing lactation (see Induced Lactation and Colactation section) and producing human milk. Breast augmentation in trans women may mask inadequate mammary tissue development or result in pressure atrophy of remaining tissue. It may also increase the risk of engorgement during induced lactation.

- Masculinizing treatments and practices: Testosterone therapy for transgender men or transmasculine people 
suppresses endogenous estrogen production that can result in atrophy of estrogen-responsive breast tissues. ${ }^{25}$ Trans men who are gestational parents generally must suspend testosterone therapy during pregnancy. This suspension may be continued in the postpartum period if lactation is desired. In addition, trans men may practice chest binding (using a tight compressive garment or bandage to flatten the appearance of the chest). This may result in compression atrophy, worsen engorgement, or lower milk supply. Finally, trans men who have undergone "top" surgery may or may not have the ability to lactate. Surgical techniques for "top" surgery vary widely from a bilateral total mastectomy with resection of the nipple areolar complex (NAC) to utilization of reduction pattern techniques with preservation of the NAC on an intact neurovascular pedicle. These techniques can result in differences in residual mammary parenchyma among individuals. Furthermore, if the nipple is completely removed and reattached, the ductal anatomy is less likely to regenerate and allow for lactation, as opposed to when it is preserved. $^{26}$ Modern NAC tattooing techniques may give the appearance of a native NAC, but the results are cosmetic only.

- Gender dysphoria as a result of lactation care: Any treatment that alters the hormonal milieu of trans patients may increase their gender dysphoria. This may be added to any suffering they experienced as a result of fertility and pregnancy care. For instance, when a trans man gestates, he will have hormonal changes from suspending testosterone therapy and from the pregnancy itself, which may be stressful, and he may have negative emotions related to his chest changes. Being sensitive to the experiences of patients means supporting them in their decision to resume genderaffirming hormonal treatments after birth, including decisions not to lactate.

\section{Fertility Options}

Reproductive options for LGBTQ+ individuals vary depending on the personal preferences of the individuals involved, their and their partner's gonads and reproductive organs, past or current therapies that may affect fertility, access to reproductive endocrinology and infertility specialists, finances/insurance coverage, and local legislation. ${ }^{27,28}$ Fertility and child-bearing goals vary greatly from individual to individual, so assumptions must be avoided. ${ }^{29-31}$ Although these can be sensitive topics and providers may be uncomfortable with their own knowledge, it is important to provide information on the possibilities and be informed about local resources and legal restrictions. Patients may be hesitant to bring up their goals or questions out of fear of discrimination or violence. ${ }^{6,29}$ As a result, parents and infants may suffer poor outcomes during this vulnerable time.

- If neither partner has child-bearing potential or neither partner desires to carry a pregnancy, their options include surrogacy (formal or informal, with sperm or egg from one or both partners or from a donor) or adoption. ${ }^{32}$

- If at least one partner has child-bearing potential (i.e., has a uterus and appropriate hormonal milieu) and desires to carry a pregnancy, they may: o conceive through penetrative (penis in vagina) intercourse, intrauterine insemination, or in vitro fertilization;

o use sperm donation (formal or informal); or

$\bigcirc$ use egg donation from a partner or donated egg.

\section{Induced Lactation and Colactation}

Some LGBTQ+ parents may desire to induce lactation, which is to start the production of milk in the absence of a pregnancy. There are many anecdotal reports and one case report of a trans woman (AMAB, on hormone replacement therapy) successfully inducing lactation. ${ }^{33}$ Regarding induced lactation in LGBTQ+ parents, we recommend the following based on our experience and the limited published information:

- Do not assume that a person with breasts will know that inducing lactation is an option, nor that they will desire to induce lactation. Rather, provide information on its possibilities and ask open-ended questions, such as, "What are your thoughts about feeding your child" and "What have you considered about inducing lactation?",

- Counsel the family about stress and maintaining mental health and relationships. ${ }^{34}$ Inducing lactation can be difficult and takes time and effort, which may increase a family's stress level. ${ }^{35}$ In addition, there is no published research on the frequency of success. Parental stress has neurodevelopmental consequences for their children, and any benefit of human milk must be weighed against this risk. ${ }^{36}$

- There exists no standard guidance on inducing lactation for any patient population. Different methods have been used for inducing lactation, including visualization/meditation, expression by hand or pump, feeding at the breast/chest, and combinations of oral contraceptive use followed by hormone withdrawal combined with galactogogues and expression (see ABM Protocol \#9 "Use of Galactogogues in Initiating or Augmenting Maternal Milk Production, Second Revision $\left.2018^{37}\right) .^{33,35,38}$

- Inducing lactation may present more of a challenge for transgender individuals. In these cases, one can generally refer to three components: hormonal priming of the mammary tissue, prolactin promotion, and hormone withdrawal with expression.

O Priming: Hormonal priming of the mammary tissue in nongestating people AFAB generally involves a combined oral contraceptive with estrogen and progesterone (e.g., ethinyl estradiol and norgestimate), taken continuously for months without a menstruation phase (placebo pills). Patients AMAB who have transitioned will likely already have a medication regimen including estrogen, progesterone, and an androgen blocker (often spironolactone) if they have not had a bilateral orchiectomy. Depending on the length of treatment, patients will have growth of mammary tissue. When patients desire to induce lactation, it has been assumed that the hormonal milieu of pregnancy should be approximated for a similar period of time as in induced lactation with nongestating people AFAB, or 
3 to 6 months. Doses of estrogen and progesterone may be increased gradually as tolerated to $8-12 \mathrm{mg}$ estrogen and $400 \mathrm{mg}$ progesterone daily during the stimulation phase as in the Reisman case report. ${ }^{33}$ Risks, especially of blood clots with high doses of estrogen, should be discussed. Laboratory testing for serum levels has been done but may or may not be useful in guiding therapy. A more useful guide may be a patient's symptoms of breast/chest changes. At this phase, many providers will recommend stimulation of the nipples and areolae and gentle hand expression one to three times per day.

o Prolactin promotion: Prolactin promotion is generally achieved with galactogogues and may be used during the hormonal stimulation phase (above) or the expression phase (below). Traditional induced lactation protocols use these medications throughout the induced lactation process, although this is not evidence based. Domperidone has the strongest evidence for successful lactation promotion, and the only published case report of successful induced lactation is in a transgender woman who used this medication. ${ }^{33}$ It is not FDA approved for use in the United States. Metoclopramide or herbal galactogogues may also be attempted. Cost, risks, and side effects of some treatments may limit a patient's ability to take these in the long term.

○ Expression: In general, withdrawal of the high doses of estrogen and progesterone during the expression phase should occur a month before a child will need to be fed. A patient may resume their original estrogen and progesterone dosages. Alternatively, as in the Reisman case report, ${ }^{33}$ estrogen may be lowered even further ( $0.025 \mathrm{mg}$ estradiol patch). Side effects of lowering estrogen to this level must be considered if a patient has been well maintained at higher levels previously. During this phase, consistent hand expression and pumping are begun, and should be gradually increased to 5-10 minutes 6-8 times per day. Flange fitting and pump teaching should be done by a trained professional. Pumping without expressing milk may be uncomfortable, and lubricants can be used in the flanges (such as lanolin, coconut oil, or lubrication sprays specific for pumping) to decrease friction. If milk is not being used, it can be frozen in capped syringes.

O If a child is being put to breast/chest during the expression phase, without or before a full supply is created, a supplemental feeding tube at the breast/ chest may be used to encourage the child to stay latched.

- Bonding should be discussed as a benefit to chestfeeding and breastfeeding. Feeding or soothing a child with one's body has been reported to be of significant value by many people with low milk production.

Some parents may consider colactation after one or more parents induce lactation, or because one parent has previously fed a prior child or is still lactating when their partner gives birth. In addition, there are families and relationships with more than two parents and, therefore, colactation may refer to milk sharing among larger groups. If a family is considering colactation:
1. Discuss expectations and goals

- Inform parents that colactation experiences are unlikely to decrease the workload for the gestational parent. This may be a misconception about colactation.

- Other duties (including chores, work/income, and infant care) should be discussed and any family/ community resources available should be identified, especially for the first 2 weeks after birth.

- Discuss parental goals for colactating. Some specific questions for parents to consider are: who will do skin-to-skin at birth, who will feed and when, and how will multiple milk supplies be maintained?

- Appendix A1 offers a sample colactation plan that can be adapted for individual parents and may be created and shared with providers before birth.

2. Support milk production and infant health

- All lactating parents will need to maintain a milk supply by feeding or expressing milk.

- Support milk production in all lactating parents with removal of milk six to eight times per day and galactogogues, as detailed in ABM Protocol $\# 9 .{ }^{37}$

- When possible, consider prioritization of colostrum feeding with the gestational parent, given the individuality of colostrum for the neonate. Colostrum is produced in the birth parent starting in the second trimester of pregnancy. ${ }^{39}$

Situations of adoption or surrogacy may be complicated by a birth taking place in a different area than the parents' home, at an unfamiliar hospital, or one with inadequate lactation support for LGBTQ+ individuals. The breastfeeding medicine provider can serve as an important mediator in this interaction. In particular, before birth:

- Consider testing for transmissible infections when inducing lactation or a nongestational parent desires to give their milk. These laboratories are routinely recommended in prenatal care and, as such, serve to guide both pregnancy and lactation management. In addition, a birth hospital may require that a parent have screening laboratories before allowing their milk to be provided. The following may be considered: HIV, syphilis, hepatitis $\mathrm{B}$ and $\mathrm{C}$ (to provide counseling in the instance of bleeding nipples/blood in the milk), and tuberculosis in high-risk areas. Cytomegalovirus testing of gestational parents and/or human milk is performed in some neonatal intensive care units (NICUs) and may be considered, especially for infants born at $<32$ weeks gestation. Donor milk banks test for Human T-lymphotropic virus types I and II (HTLV I and II) and may be considered, especially in endemic areas. ${ }^{40}$ Although these are medically appropriate suggestions, it may be considered offensive to parents who may feel this is stigmatizing. However, nongestating lactating parents may be reassured to know that these are routine screenings for gestational parents and milk donors.

- The parent's breasts/chest should be examined for herpes, and the parent should be counseled about not expressing or feeding during a herpes outbreak on the nipple(s), areola, or skin that would come into contact with the flanges of the pump or hangs if hand expressing. 
- With patient consent, contact the lactation consultant or breastfeeding support person(s) at the birth hospital; this is likely to encourage accurate pronoun and name use, discuss milk status of the parent providing milk, and discuss assistance for feeding at the breast/chest if this is desired by the parent (i.e., supplemental feeding tubes at the breast/chest, supplementation, etc.)

\section{Birth, Birth Plans, and Breast/Chestfeeding}

The birth experience for LGBTQ+ parents may be socially complex if the birth facility is not prepared to work with families with gender and sexual diversities. In addition, families may have experienced discrimination, substandard care, and/or trauma in the health care setting. ${ }^{6-8,41}$ In these cases, the lactation support team has the unique opportunity to be prepared, respectful, and kind.

- Regardless of a family's prior experience, families with LGBTQ+ members should be provided appropriate standard care to promote human milk feeding, including prenatal education, skin-to-skin immediately after birth, rooming in, feeding on demand, and appropriate follow-up (see ABM Clinical Protocol \#5). ${ }^{42}$ Standard guidance on exclusive breastfeeding may need to be altered based on the expected production of a nongestational parent and whether milk will be provided by a gestational parent. For instance, full production is not usually achievable in induced lactation, or after "top" surgery. Therefore, parents should be encouraged to provide as much human milk as possible, and be educated on the indications for supplementation, types and methods of supplementation, and need for close follow-up.

- If two or more family members plan to breast/ chestfeed:

- Cocreate a plan surrounding feeding preferences

- Determine which parent will do skin-to-skin and who will feed in the hospital, with support of all milk supplies (Appendix A1).

$\bigcirc$ All lactating parents should have medications and health conditions reviewed for any possible impacts on lactation or infant health.

- Privacy may be a concern. Providers and lactation consultants should be aware that some transgender and gender-expansive patients may have body dysphoria, surgical scars, or experiences of trauma, abuse, and violence. Privacy during examination, feeding, and latch assistance may be very important to the family and should be respected. Also, a hands-off approach for lactation assistance should be considered if preferred by the family.

- Latch considerations include working with the areolar/chest contour, flexibility of the chest/breast, positioning, and assistive devices:

○ For trans men who have had "top" surgery, or trans women who have had breast augmentation, the mammary tissue may be less flexible and areolae may be flat. If latch is desired and the infant is not able to latch, a nipple shield may be considered. Some nipple shields have a nipple similar to a bottle nipple, which may further aid in latch, especially if the tissue is not flexible and cannot be pulled into a standard shield.

O Positioning of infants for trans men who have had "top" surgery may also be higher on the parent's body, as there will generally be no pendulousness to the chest. Additional pillows may be needed.

○ For any patient with latch difficulties, another approach is to form the breast/chest tissue with hands to encourage infant latch. As with any patient, a hands-off or hand-over-hand approach is most effective for teaching and preferred by most patients.

○ For patients with low milk supply, a supplemental feeding tube attached to a bottle, syringe, or specific supplemental device with human milk or artificial human milk substitute (infant formula) may be applied to provide nutrition to the infant at the same time as stimulation and milk transfer from the parent. The end of the feeding tube should reach the nipple tip, and the tube may be secured by tape to the chest wall. Such a tube may be used inside or outside of a nipple shield. There is no published evidence on the best methods of use for these devices.

\section{Additional Considerations in the Hospital and NICU}

LGBTQ+ families face special challenges in the intensive care unit setting. First, there is extensive staff, which extends beyond the primary team of providers and nurses. This, with frequent changes of staff, makes it more difficult to ensure all staff involved are aware of familial relationships and affirmed pronouns/names. Furthermore, many NICUs are in educational facilities, exposing LGBTQ+ parents to medical students, residents, and fellows with varying levels of training in culturally competent health care. Based on preliminary qualitative research with LGBTQ+ parents, interactions with staff play a major role in the overall parental experience. These interactions begin with reception and continue through discharge.* As already described but accentuated by multipatient rooms, loss of privacy may add another barrier to breast/chestfeeding for parents with gender dysphoria or histories of trauma or abuse.

In addition, LGBTQ+ families face the same barriers as others attempting to breast/chestfeed in the NICU, including the reliance on breast pumps to initiate and maintain supply, difficulty establishing skin-to-skin due to equipment needed for the infant (i.e., endotracheal tubes, mechanical ventilators, and central lines), difficulty feeding a premature infant with poor feeding cues and oromotor skills, and the many competing interests of living with a hospitalized child (financial, family, support, emotional, employment, transportation, etc.).

The following may be helpful for families identifying as LGBTQ+ in the NICU, as well as any institutional setting:

- Ensure that check-in forms use inclusive language (e.g., fields for "parent 1, parent 2," instead of "mother, father").

\footnotetext{
*Jackson J, Dadiz R. (2020). LGBTQI Parent Experience with Newborn Nutrition. Unpublished raw data.
} 
- Ensure that leadership emphasizes the need for inclusive and respectful language at all times. This will often include the need for prior training, clear hand-off between shifts involving different caregivers, and the lack of tolerance for any disrespectful behavior.

- Use skills of introduction, asking pronouns, asking for which words the family would like to use for parenting, feeding, etc., and ensuring that these preferences are clearly passed off between caregivers so that the burden of repetitive education is not placed on families.

- Ensure that parental concerns are addressed and that all parents are included in medical decision-making. ${ }^{8,41}$

\section{Donor Milk and Informal Milk Sharing}

Multiple sources confirm donor human milk is a safe and effective source of nutrition for the extremely preterm, very low birth weight, or medically complex infant. ${ }^{43,44} \mathrm{Un}$ fortunately, in many countries, donor milk is not available, is only available for high-risk infants, or is prohibitively expensive. Thus, LGBTQ+ families are at a disadvantage when they are unable to produce their own human milk. Because of this, informal milk sharing communities are popular among LGBTQ+ parents. ${ }^{45}$ The American Academy of Pediatrics (AAP) does not endorse the use of informal milk sharing due to the inability to assess risk. ${ }^{43}$ The ABM, however, acknowledges the risks and benefits of informal milk sharing, and suggests medical screening of the donor and safe milk handling practices to maximize safety. ${ }^{46}$ Both the AAP and $\mathrm{ABM}$ recommend against Internet-based human milk sharing.

\section{Supports}

Few but growing numbers of resource exist to support LGBTQ+ families and their health care providers during pregnancy and lactation. Unfortunately, resources on parenting and lactation remain hetero- and cisnormative with an explicit maternal focus when discussing breastfeeding and lactation. They do not tend to discuss or include other types of families and parents. LGBTQ+ parents have described a negative impression of breastfeeding books and phone apps because of gender normativity.

- Written resource including lactation:

○ Where's the Mother?: Stories from a Transgender Dad by Trevor MacDonald ${ }^{3}$

- Online supports

- Increasing use of Facebook and social media groups for support

$\square$ Online milk sharing communities (i.e., MilkShare, Eats on Feets)

$\square$ "Birthing and Breast or Chestfeeding Trans People and Allies Facebook Group"

$\square$ Helpful searches may include "trans" or "LGBTQ" and "breastfeeding" or "parenting"

$\circ$ Internet mailing list for lesbian, bisexual, and transgender mothers, see Lev et al., 2005. ${ }^{47}$

$\circ$ Breastfeeding and parenting from a transgender perspective (milkjunkies.net).

- Health care providers

○ GOLD learning online: Jaye Simpson "LGBTQ Parents and Lactation-An Exploration in LGBTQ
Culture" available at: https://www.goldlearning .com/lecture/221.

- Professional organizations:

○ World Professional Association for Transgender Health.

○ La Leche League International, https://www.llli.org/ breastfeeding-info/transgender-non-binary-parents/.

- Group meetings:

O Peer support is an important predictor of a parent's success attaining their personal breastfeeding goals. ${ }^{48}$ Trans parents and other LGBTQ+ families may already feel isolated, especially if they do not know other LGBTQ+ families. ${ }^{28}$

\section{Future Research}

Significant research gaps exist in this field. In the future, it is unlikely that grouping sexual orientation and gender identity will provide appropriate research or practical guidance. It is, therefore, suggested that future research examine LGBTQ+ community subgroups. Specifically, investigation is needed into milk production in parents who have undergone gender-affirming surgeries and those who have induced lactation. Given that this is a new and growing field and the history of neglect and discrimination in the LGBTQ+ community, community-based participatory research and qualitative studies informing future research and support should be strongly considered.

\section{Disclosure Statement}

C.B.R.-C. is a consultant for May \& Meadow, a biotech start-up. No competing financial interests exist for the remaining authors.

\section{Funding Information}

No funding was received for the creation of this protocol.

\section{References}

1. Terminology and Definitions. UCSF transgender care. 2016. Available at https://transcare.ucsf.edu/guidelines/ terminology (accessed November 20, 2019).

2. Glossary of LGBT terms for Health Care Teams. National LGBT Health Education Center. Fenway Institute. 2017. Available at https://www.lgbthealtheducation.org/wpcontent/uploads/2018/03/Glossary-2018-English-update-1 .pdf (accessed November 20, 2019).

3. MacDonald T. Where's the Mother? Stories from a Transgender Dad. Trans Canada Press, 2016.

4. World Health Organization, 2018. The ICD-11 for mortality and morbidity statistics. Available at https://icd.who.int/ browse11/1-m/en (accessed April 2, 2020).

5. Tasker F. Lesbian mothers, gay fathers, and their children: A review. J Dev Behav Pediatr 2005;26:224-240.

6. James SE, Herman JL, Rankin S, et al. Report of the 2015 U.S.Transgender Survey. Washington, DC: National Center for Transgender Equality, 2016. Available at https:// transequality.org/sites/default/files/docs/usts/USTS-FullReport-Dec17.pdf (accessed April 2, 2020).

7. Daniel H, Butkus R; Health and Public Policy Committee of American College of Physicians. Lesbian, gay, bisex- 
ual, and transgender health disparities: Executive summary of a policy position paper from the American College of Physicians. Ann Intern Med 2015;163:135137.

8. Wells MB, Lang SN. Supporting same-sex mothers in the Nordic child health field: A systematic literature review and meta-synthesis of the most gender equal countries. J Clin Nurs 2016;25:3469-3483.

9. Grant JM, Lisa AM, Justin T, et al. Injustice at Every Turn: A Report of the National Transgender Discrimination Survey. Washington: National Center for Transgender Equality and National Gay and Lesbian Task Force, 2011.

10. Davis K. Southern Comfort. HBO Theatrical Documentary Presentations. Released 2001 at the Film Forum, New York, NY. Film. Available at https://tubitv.com/movies /319922/southern_comfort?utm_source=google-feed\& tracking=google-feed (accessed September 9, 2019).

11. Braun H, Nash R, Tangpricha V, et al. Cancer in transgender people: Evidence and methodological considerations. Epidemiol Rev 2017;39:93-107.

12. Abbott TB. SOUTHERN COMFORT. Queer Stud Media Pop Culture 2018;3:403+.

13. Fernandez ME. Death Suit Costs City $\$ 2.9$ million. Washington Post. 1998. Available at https://www.washing tonpost.com/archive/local/1998/12/12/death-suit-costs-city29-million/b8ab4d34-1907-463c-b5d5-64ec00dee2a1/ (accessed December 1, 2019).

14. Makadon HJ, Mayer KH, Potter J, Goldhammer H, eds. The Fenway Guide to Lesbian, Gay, Bisexual, and Transgender Health, 2nd Edition. Philadelphia: American College of Physicians, 2015.

15. Chetwynd EM, Facelli V. Lactation support for LGBTQ+ families. J Hum Lact 2019;35:244-247.

16. Rushin S, Carroll J. Bathroom Laws as Status Crimes, 86 Fordham L. Rev 1. 2017. Available at: https://ir.lawnet .fordham.edu/flr/vol86/iss1/11 (accessed April 2, 2020).

17. Juntereal NA, Spatz DL. Same-sex mothers and lactation. MCN Am J Matern Child Nurs 2019;44:164-169.

18. MacDonald TK. Lactation care for transgender and nonbinary patients: Empowering clients and avoiding aversives. J Hum Lact 2019;35:223-226.

19. Finlayson C, Johnson EK, Chen D, et al. Proceedings of the working group session on fertility preservation for individuals with gender and sex diversity. Transgender Health 2016;1:99-107.

20. Moravek MB. Fertility preservation options for transgender and gender-nonconforming individuals. Curr Opin Obstet Gynecol 2019;31:170-176.

21. Abramowitz J, Tangpricha V. Hormonal management for transfeminine individuals. Clin Plast Surg 2018;45:313317.

22. Maxwell S, Noyes N, Keefe D, et al. Pregnancy outcomes after fertility preservation in transgender men. Obstet $G y$ necol 2017;129:1031-1034.

23. Ellis SA, Dalke L. Midwifery care for transfeminine individuals. J Midwifery Womens Health 2019;64:298311.

24. Wierckx K, Gooren L, T'Sjoen G. Clinical review: Breast development in trans women receiving cross-sex hormones. J Sex Med 2014;11:1240-1247.

25. T'Sjoen G, Arcelus J, Gooren L, et al. Endocrinology of transgender medicine. Endocr Rev 2019;40:97-117.
26. Kraut RY, Brown E, Korownyk C, et al. The impact of breast reduction surgery on breastfeeding: Systematic review of observational studies. PLoS One 2017;12: e0186591.

27. Kim HH. Family building by same-sex male couples via gestational surrogacy. Semin Reprod Med 2017;35:408414.

28. Light AD, Obedin-Maliver J, Sevelius JM, et al. Transgender men who experienced pregnancy after female-tomale gender transitioning. Obstet Gynecol 2014;124:1120 1127.

29. Hoffkling A, Obedin-Maliver J, Sevelius J. From erasure to opportunity: A qualitative study of the experiences of transgender men around pregnancy and recommendations for providers. BMC Pregnancy Childbirth 2017; 17(Suppl 2):332.

30. MacDonald T, Noel-Weiss J, West D, et al. Transmasculine individuals' experiences with lactation, chestfeeding, and gender identity: A qualitative study. BMC Pregnancy Childbirth 2016;16:106.

31. Wolfe-Roubatis E, Spatz DL. Transgender men and lactation: What nurses need to know. MCN Am J Matern Child Nurs 2015;40:32-38.

32. Grover SA, Shmorgun Z, Moskovtsev SI, et al. Assisted reproduction in a cohort of same-sex male couples and single men. Reprod Biomed Online 2013;27:217-221.

33. Reisman T, Goldstein Z. Case report: Induced lactation in a transgender woman. Transgend Health 2018;3: 24-26.

34. Appelgren Engström H, Häggström-Nordin E, Borneskog $\mathrm{C}$, et al. Mothers in same-sex relationships describe the process of forming a family as a stressful journey in a heteronormative world: A Swedish grounded theory study. Matern Child Health J 2018;22:1444-1450.

35. Wilson E, Perrin MT, Fogleman A, et al. The intricacies of induced lactation for same-sex mothers of an adopted child. J Hum Lact 2015;31:64-67.

36. Koutra K, Chatzi L, Bagkeris M, et al. Antenatal and postnatal maternal mental health as determinants of infant neurodevelopment at 18 months of age in a mother-child cohort (Rhea Study) in Crete, Greece. Soc Psychiatry Psychiatr Epidemiol 2013;48:1335-1345.

37. Brodribb W. ABM Clinical Protocol \#9: Use of galactogogues in initiating or augmenting maternal milk production, second revision 2018. Breastfeed Med 2018;13:307314.

38. Szucs KA, Axline SE, Rosenman MB. Induced lactation and exclusive breast milk feeding of adopted premature twins. J Hum Lact 2010;26:309-313.

39. Sriraman NK. The nuts and bolts of breastfeeding: Anatomy and physiology of lactation. Curr Probl Pediatr Adolesc Health Care 2017;47:305-310.

40. Prendergast AJ, Goga AE, Waitt C, et al. Transmission of CMV, HTLV-1, and HIV through breastmilk. Lancet Child Adolesc Health 2019;3:264-273.

41. Andersen AE, Moberg C, Bengtsson Tops A, et al. Lesbian, gay and bisexual parents' experiences of nurses' attitudes in child health care-A qualitative study. J Clin Nurs 2017; 26:5065-5071.

42. Holmes A, McLeod AY, Bunik M. ABM clinical protocol \#5: Peripartum breastfeeding management for the healthy mother and infant at term, revision 2013. Breastfeed Med 2013;8:469-473. 
43. Committee on Nutrition; Section on Breastfeeding; Committee on Fetus and Newborn. Donor human milk for the high-risk infant: Preparation, safety, and usage options in the United States. Pediatrics 2017;139:pii: e20163440.

44. Coutsoudis I, Adhikari M, Nair N, et al. Feasibility and safety of setting up a donor breastmilk bank in a neonatal prem unit in a resource limited setting: An observational, longitudinal cohort study. BMC Public Health 2011;11: 356.

45. Palmquist AE, Doehler K. Human milk sharing practices in the U.S. Matern Child Nutr. 2016;12:278-290.

46. Sriraman NK, Evans AE, Lawrence R, et al. Academy of Breastfeeding Medicine's 2017 position statement on informal breast milk sharing for the term healthy infant. Breastfeed Med 2018;13:2-4.

47. Lev AI, Dean G, DeFilippis L, et al. Dykes and tykes: A virtual lesbian parenting community. J Lesbian Stud 2005; 9:81-94.

48. Shakya P, Kunieda MK, Koyama M, et al. Effectiveness of community-based peer support for mothers to improve their breastfeeding practices: A systematic review and metaanalysis. PLoS One 2017;12:e177434.

ABM protocols expire 5 years from the date of publication. Content of this protocol is up-to-date at the time of publication. Evidence-based revisions are made within 5 years or sooner if there are significant changes in the evidence.

Rita Lynne Ferri, BA, lead author Casey Braitsch Rosen-Carole, MD, MPH, MEd Jason Jackson, DO Elizabeth Carreno-Rijo, $M D, M P H$ Katherine Blumoff Greenberg, MD
The Academy of Breastfeeding Medicine Protocol Committee:

Michal Young, MD, FABM, Chairperson Larry Noble, MD, FABM, Translations Chairperson Melissa Bartick, MD, MSc, FABM

Sarah Calhoun, $M D$

Monica V. Carceles-Fraguas, MD, IBCLC, FABM

Megan Elliott-Rudder, $M D$

Lori Feldman-Winter, MD, MPH

Laura Rachael Kair, MD, FABM

Susan Lappin, $M D$

Ilse Larson, $M D$

Ruth A. Lawrence, MD, FABM

Yvonne Lefort, MD, FABM

Kathleen A. Marinelli, MD, FABM

Nicole Marshall, MD, MCR

Katrina Mitchell, MD, FABM

Catherine Murak, MD

Eliza Myers, $M D$

Monica Pina, MD

Sarah Reece-Stremtan, $M D$

Susan Rothenberg, MD, IBCLC, FABM

Tricia Schmidt, $M D$

Tomoko Seo, MD, FABM

Christina Smillie, MD, FAAP, IBCLC, FABM

Natasha Sriraman, $M D$

Elizabeth K. Stehel, MD

Gina Weissman, DMD, RN, IBCLC, FABM

Nancy Wight, $M D$

Adora Wonodi, MD

Deena Zimmerman, MD, MPH, IBCLC

For correspondence: abm@bfmed.org

\section{Appendix A1. Colactation Feeding Plan}

(Please note, this feeding plan is filled out by the gestational parent. In a hospital or institution, this would be the patient who would give their consent for health care choices, in most cases. It assumes a two-person parenting relationship, which may not be applicable. It may, therefore, need to be adapted to specific circumstances.)

Date:

\section{Colactation Infant Feeding Plan}

My name is and my goal is to cofeed my baby with my (partner status) (name)

The benefits of human milk feeding are very important to us and our baby. We would like to have our guidelines supported as long as it is medically safe. We also would like to ensure that my milk supply is well established, while supporting in learning how to nurse and bond with our new baby. Because of this, some things in our feeding plan are the same as other peoples, and some are different.

\section{ROUTINE (CHECK ALL THAT APPLY):}

$\square$ Skin-to-skin: please place our baby skin-to-skin on my chest after delivery. Please do checkups and procedures on our baby while they are skin-to-skin, when possible.

$\square$ Emergency Cesarean: if I am unable to hold the baby skin-to-skin at birth, please allow my (partner status) (name)

to do so and latch for the first time.

$\square$ Exclusive breastfeeding: our goal is to exclusively breastfeed our baby. Please do not give my baby any formula before speaking to us about it.

$\square$ No bottles or pacifiers: please do not give pacifiers or bottles without speaking with us first.

$\square$ Feed on cue: please help me to learn the signs that my baby is hungry and feed my baby when they are ready to eat.

$\square$ Rooming in: please help our baby and I stay in our room together 24 hours per day. 
FOR COLACTATION (CHECK ALL THAT APPLY):

$\square$ At the time of delivery, my (partner status) (name) making _ mL per day of milk.

$\square$ Initial skin-to-skin and latch will be done by if we are medically able.

$\square$ If I am not available after birth to do skin-to-skin and latch, please allow my (name)__ to do so and latch for the first time.

$\square$ After the first latch, we would like to primarily feed the baby at the breast.

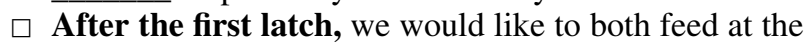
breast. We know that if we do this, whoever is not feeding at the breast will need to hand express or pump milk, and this may result in a decreased milk supply. We also understand that if my (partner status)
, (name)

is

not making milk, a supplemental nursing system will need to be used. In all cases, baby's weight will need to be closely monitored.

$\square$ Please provide my (partner status) (name)

help with a supplemental nursing system while in the hospital.

$\square$ After we go home, we would like to

This plan has been discussed with my provider:

My baby's provider/pediatrician will be:

Discuss with baby's provider/pediatrician 